Purdue University

Purdue e-Pubs

6-6-2008

\title{
Nonequilibrium Green's function treatment of phonon scattering in carbon nanotube transistors
}

Siyuranga O. Koswatta

Purdue University - Main Campus, koswatta@purdue.edu

Follow this and additional works at: http://docs.lib.purdue.edu/nanodocs

Koswatta, Siyuranga O., "Nonequilibrium Green's function treatment of phonon scattering in carbon nanotube transistors" (2008).

Other Nanotechnology Publications. Paper 83.

http://docs.lib.purdue.edu/nanodocs/83

This document has been made available through Purdue e-Pubs, a service of the Purdue University Libraries. Please contact epubs@purdue.edu for additional information. 


\title{
Nonequilibrium Green's Function Treatment of Phonon Scattering in Carbon-Nanotube Transistors
}

\author{
Siyuranga O. Koswatta, Sayed Hasan, Mark S. Lundstrom, Fellow, IEEE, \\ M. P. Anantram, and Dmitri E. Nikonov, Senior Member, IEEE
}

\begin{abstract}
We present a detailed treatment of dissipative quantum transport in carbon-nanotube field-effect transistors (CNTFETs) using the nonequilibrium Green's function formalism. The effect of phonon scattering on the device characteristics of CNTFETs is explored using extensive numerical simulation. Both intraand intervalley scattering mediated by acoustic (AP), optical (OP), and radial-breathing-mode (RBM) phonons are treated. Realistic phonon dispersion calculations are performed using forceconstant methods, and electron-phonon coupling is determined through microscopic theory. Specific simulation results are presented for $(16,0),(19,0)$, and $(22,0)$ zigzag CNTFETs, which are in the experimentally useful diameter range. We find that the effect of phonon scattering on device performance has a distinct bias dependence. Up to moderate gate biases, the influence of high-energy OP scattering is suppressed, and the device current is reduced due to elastic backscattering by AP and low-energy RBM phonons. At large gate biases, the current degradation is mainly due to high-energy OP scattering. The influence of both AP and high-energy OP scattering is reduced for larger diameter tubes. The effect of RBM mode, however, is nearly independent of the diameter for the tubes studied here.
\end{abstract}

Index Terms-Carbon nanotube, dissipative transport, nonequilibrium Green's function (NEGF), phonon scattering, quantum transport, transistor.

\section{INTRODUCTION}

$\mathbf{S}$ INCE THE first demonstration of carbon-nanotube (CNT) field-effect transistors in 1998 [1], [2], there has been tremendous progress in their performance and physical understanding [3]. Both electronic and optoelectronic devices based on CNTs have been realized, and the fabrication processes have been optimized. Ballistic transport in CNTs has been experimentally demonstrated for low-bias conditions at low temperatures [4], [5]. High-performance CNT transistors op-

Manuscript received January 3, 2007; revised May 29, 2007. This work was supported in part by the NASA Institute for Nanoelectronics and Computing (NASA INAC NCC 2-1363), by the NASA Contract NAS2-03144 to UARC, and by the Intel Corporation. The review of this paper was arranged by Editor S. Takagi.

S. O. Koswatta and M. S. Lundstrom are with the School of Electrical and Computer Engineering, Purdue University, West Lafayette, IN 47907 USA (e-mail: koswatta@purdue.edu).

S. Hasan is with the Silicon Technology Development Group, Texas Instruments, Dallas, TX 75243 USA (e-mail: shasan@ti.com).

M. P. Anantram is with the Nanotechnology Engineering Group, Department of Electrical and Computer Engineering, University of Waterloo, Waterloo, ON N2L 3G1, Canada.

D. E. Nikonov is with the Technology and Manufacturing Group, Intel Corporation, SC1-05, Santa Clara, CA 95052 USA (e-mail: dmitri.e.nikonov@ intel.com).

Color versions of one or more of the figures in this paper are available online at http://ieeexplore.ieee.org.

Digital Object Identifier 10.1109/TED.2007.902900 erating close to the ballistic limit have also been reported [6]-[8]. The experimentally obtained carrier mobilities are on the order of $10^{4} \mathrm{~cm}^{2} / \mathrm{V} \cdot \mathrm{s}$ [9], [10], so exceptional device characteristics can indeed be expected. Current transport in long metallic CNTs, however, is found to saturate at $\sim 25 \mu \mathrm{A}$ at high biases, and the saturation mechanism is attributed to phonon scattering [11]. On the other hand, for short length metallic tubes, the current is found not to saturate but to increase well beyond the above limit [12], [13]. Nevertheless, carrier transport in these shorter tubes is still influenced by phonon scattering and warrants a detailed physical understanding of the scattering mechanisms due to its implications on device characteristics for both metallic and semiconducting CNTs.

There have been many theoretical studies on the calculation of carrier scattering rates and mobilities in CNTs using semiclassical transport simulation based on the Boltzmann equation [14]-[20]. Similarly, phonon-mode calculations for CNTs have also been performed with varying degrees of complexity: continuum and force-constant models [21]-[23] to first-principle-based methods [24]-[26]. The determination of electron-phonon (e-ph) coupling strength is performed using tight-binding calculations [27]-[29] as well as first-principle techniques [30]. It has been shown, however, that the influence of phonon scattering on device performance depends not only on the phonon modes and e-ph coupling but also on the device geometry [31], [32]. Therefore, in order to ascertain the impact of phonon scattering on device performance, the aforementioned calculations should be done in the context of specific device geometry. To that end, the phonon scattering in CNT transistors has been treated using the semiclassical Boltzmann transport to determine its effects on device characteristics [31], [33]. Semiclassical transport, however, can fail to rigorously treat important quantum-mechanical effects, such as band-to-band tunneling, that have been deemed important in these devices [34]-[36]. Therefore, a device simulator based on dissipative quantum transport that rigorously treats the effects of phonon scattering will be essential in properly assessing the CNT transistor characteristics and in gaining a deeper understanding of carrier transport at the nanoscale.

The nonequilibrium Green's function (NEGF) formalism has been employed to describe the dissipative quantum transport in nanoscale devices [37]-[39]. Diverse structures for the conducting channel required nontrivial variations on the NEGF approaches to the dissipative quantum transport. Phonon scattering has been studied in resonant tunneling devices [40], [41], in molecules [42], and in organic nanostructures [43]. The effect of e-ph interaction in silicon nanowire transistors in the 
effective mass approximation has been studied in [44] and [45]. The NEGF formalism has also been used in treating the effects of phonon scattering in CNT Schottky barrier transistors [46], [47]. It has been successfully used in investigating the impact of phonon scattering and in exploring interesting transport mechanisms such as phonon-assisted inelastic tunneling in CNT metal-oxide-semiconductor field-effect transistors (MOSFETs) with doped source and drain contacts (hereafter, simply referred to as CNTFETs) [32], [34]-[36]. The NEGF simulation of ballistic transport in CNTFETs is reported in [48]. Here, we extend the previous work [48] and present the detailed simulation technique employed for the treatment of phonon scattering in them.

The treatment of quantum transport in this paper is based on the atomistic-scale tight-binding description [21]. Unlike the effective mass approximation, the bandstructure in this approach is not taken from empirical data, but it is obtained from the structure's Hamiltonian, including subbands of both valence and conduction bands. In addition, the band-to-band tunneling (which limits the OFF-current for CNTFETs) is naturally obtained from this approach but would be absent in the effective mass approach. The objective of this paper is to give a comprehensive description of the method used in [32], [34][36] and to discuss the results of the simulation in greater detail. The rest of this paper is organized as follows. Section II describes the tight-binding scheme, the self-consistent electrostatics, and the treatment of e-ph coupling for NEGF modeling of the CNTFETs. Section III summarizes the numerical procedures used for the simulation of phonon scattering in the self-consistent Born approximation. Section IV, followed by the conclusion in Section V, has detailed simulation results and discusses the impact of phonon scattering on CNTFET characteristics. It compares the diameter dependence of the effect of phonon scattering in $(16,0),(19,0)$, and $(22,0)$ zigzag CNTs [i.e., $\bmod (n-m, 3)=1$ type], which are in the experimentally useful diameter range $(1.2-1.8 \mathrm{~nm})$ below which the contact properties degrade and above which the bandgap is too small for useful operation [49].

\section{METHOD}

\section{A. Treatment of Transport by NEGF}

A detailed description of the NEGF modeling of ballistic transport in CNTFETs is described in [48]. Here, we present a brief overview of that device model for the sake of completeness. The device Hamiltonian used in this paper is based on the atomistic nearest neighbor $p_{z}$-orbital tight-binding approximation [21]. The device geometry, which is shown in Fig. 1(a), is a CNT MOSFET with doped source and drain regions $\left(L_{\mathrm{SD}}\right)$ and a cylindrical wrap-around metallic gate electrode over the intrinsic channel region $\left(L_{\mathrm{ch}}\right)$. The gate oxide with thickness $t_{\text {OX }}$ covers the full length of the tube. We employ artificial heavily doped extension regions $L_{\text {ext }}$. They do not influence the transport in the working part of the transistor but are useful for better numerical convergence purposes when phonon scattering is present (however, they are not necessary for ballistic simulations). The cylindrical geometry of this device ensures symmetry in the angular direction, thus drastically simplifying

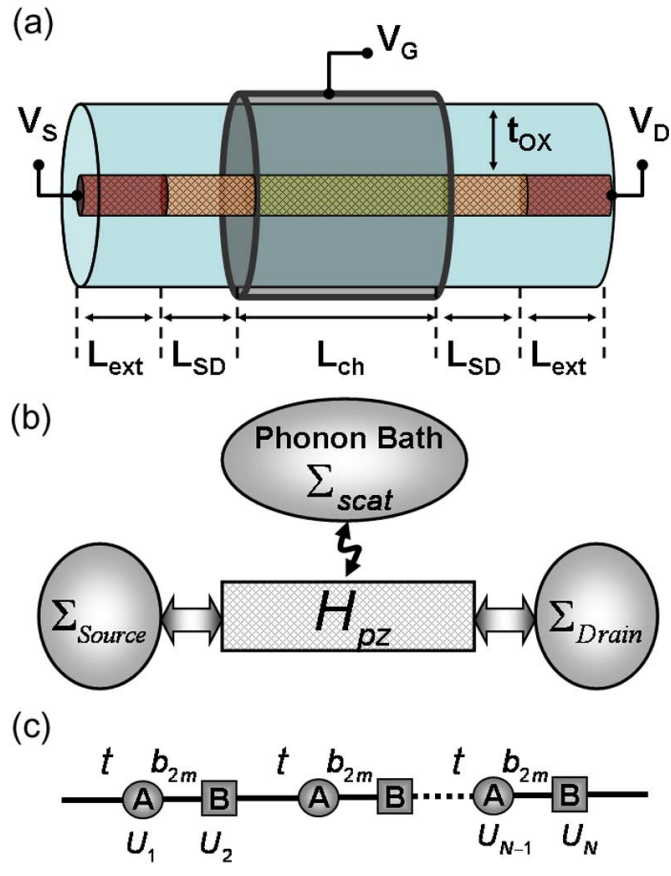

Fig. 1. (a) Device structure with wrap-around gate. (b) NEGF model with coupling to the phonon bath. (c) Mode-space Hamiltonian.

the mode-space treatment of electron transport [48], [50]. It also permits the treatment of self-consistent electrostatics using a 2D finite difference method [48]. The source and drain electrodes are treated as quasi-continuum reservoirs in thermal equilibrium and are modeled by the contact self-energy functions as in [48].

Note that, in this paper, we use the notation convention for the NEGF method which is more intuitive for device applications and is based on Datta's book [37]. Another is traditional in condensed matter physics and is exemplified by [38]. The conversion between these equivalent notations is specified in [57, Appendix A].

The NEGF model of the CNTFET used for transport simulations is shown in Fig. 1(b). Here, $H_{\mathrm{pz}}$ is the device Hamiltonian, and the self-energy functions $\Sigma_{\mathrm{S} / \mathrm{D}}$ represent the semiinfinite ideal source/drain contacts. $\Sigma_{\text {scat }}$ is the self-energy for the e-ph interaction, and one sets $\Sigma_{\text {scat }}=0$ for the ballistic approximation. A detailed specification of $\Sigma_{\text {scat }}$ is presented later in Section II-D. Finally, the retarded Green's function for the device in matrix form is given by [37]

$$
G(E)=\left[\left(E+i \eta^{+}\right) I-H_{\mathrm{pz}}-\Sigma(E)\right]^{-1}
$$

where $\eta^{+}$is an infinitesimal positive value, and $I$ is the identity matrix [37]. The self-energy contains contributions from all mechanisms of relaxation, which are the source and drain electrodes, and from scattering [37]

$$
\Sigma(E)=\Sigma_{S}(E)+\Sigma_{D}(E)+\Sigma_{\text {scat }}(E) .
$$

Note that, in (2), the self-energy functions are, in general, energy dependent.

In the mode-space treatment of an $(n, 0)$ zigzag CNT, the dependence of the electronic state on the angle along the tube's 
circumference $\varphi$ is expanded in a set of circular harmonics $\exp (i m \varphi)$ with the angular quantum number $m$. It spans the integer values of 1 to $2 n$ or, equivalently, $-n+1$ to $n$. The integer values of $m$ outside this range would produce equivalent harmonics at the crystal lattice sites. The total Hamiltonian splits into independent matrices for subbands associated with each value of $m$ [48], giving rise to a 1-D Hamiltonian with two-site unit cell, as schematically shown in Fig. 1(c), where each site corresponds to one of the two nonequivalent realspace carbon rings, $\mathrm{A}$ or $\mathrm{B}$. The period of the zigzag tube in the longitudinal direction contains four such rings, $\mathrm{ABAB}$, and has a length of $3 a_{\mathrm{cc}}$ [21], where $a_{\mathrm{cc}}=0.142 \mathrm{~nm}$ is the carbon-carbon bond length in graphene. Therefore, the average distance between rings is

$$
\Delta z=\frac{3 a_{\mathrm{cc}}}{4} .
$$

The diameter of the zigzag nanotube is [21]

$$
d_{t}=\frac{n \sqrt{3} a_{\mathrm{cc}}}{\pi} .
$$

The mode-space transformation procedure of the real-space atomistic tight-binding Hamiltonian is well described in [48], and it is not repeated here. The two-site unit cell, as expected, gives rise to the two subbands corresponding to the conduction and valence bands. The Hamiltonian matrix for the subbands with angular quantum number $m$ in an $(n, 0)$ zigzag CNT is then given by [48]

$$
H_{\mathrm{pz}}=\left[\begin{array}{cccccc}
U_{1} & b_{2 m} & & & & \\
b_{2 m} & U_{2} & t & & 0 & \\
& t & U_{3} & b_{2 m} & & \\
& & & \ddots & & \\
& 0 & & t & U_{N-1} & b_{2 m} \\
& & & & b_{2 m} & U_{N}
\end{array}\right]_{N \times N}
$$

where $b_{2 m}=2 t \cos (\pi m / n), t \approx 3 \mathrm{eV}$ is the nearest neighbor hopping parameter, and $N$ is the total number of carbon rings along the device. Here, the diagonal elements $U_{j}$ correspond to the on-site electrostatic potential along the tube surface. All electronic subbands in a CNT are fourfold degenerate: due to the two spin states and the twofold valley degeneracy [21]. The valley degeneracy comes from the two subbands with the same energy dispersion but with different $m$-values. Each subband can be represented as a cut of the graphene 2-D Brillouin zone by a line with a constant momentum $k_{y}$. In this paper, we equate momentum with wavevector, having the dimension of inverse length. The cuts closest to the $K$-points of graphene correspond to the lowest energy conduction subbands as well as the highest energy valence subbands and correspond in zigzag tubes to angular momenta $m_{L 1}=\operatorname{round}(2 n / 3)$ and $m_{L 2}=$ $\operatorname{round}(4 n / 3)$.

Level broadening can be defined as follows[37]:

$$
\Gamma(E) \equiv i\left[\Sigma(E)-\Sigma^{\dagger}(E)\right]=\Sigma^{\text {in }}(E)+\Sigma^{\text {out }}(E)
$$

where $\Sigma^{\dagger}$ represents the Hermitean conjugate of $\Sigma$ matrix defined by (2). Here, $\Sigma^{\text {in/out }}$ are the in/out-scattering functions [see (9) and (10)]. The same relations apply separately to each mechanism of relaxation. For a layered structure like the CNT, the source self-energy function $\Sigma_{S}$ has all its entries zero except for the $(1,1)$ element, i.e. [48],

$$
\Sigma_{S}(i \neq 1, j \neq 1)=0
$$

and

$$
\begin{aligned}
\Sigma_{S}(1,1) & =\alpha_{\text {source }}-\sqrt{\alpha_{\text {source }}^{2}-t^{2}} \\
\alpha_{\text {source }} & =\frac{\left(E-U_{1}\right)^{2}+t^{2}-b_{2 m}^{2}}{2\left(E-U_{1}\right)} .
\end{aligned}
$$

Similarly, $\Sigma_{D}$ has only its $(N, N)$ element nonzero, and it is given by equations similar to (7) and (8) with $U_{1}$ replaced by $U_{N}$. As mentioned earlier, $\Sigma_{\mathrm{S} / \mathrm{D}}$ self-energies rigorously capture the effect of semiinfinite contacts on the device. The presence of e-ph scattering modifies the contact self-energies, as described, e.g., in [51] and [52]. With this, we can define the in/out-scattering functions for contact coupling

$$
\begin{aligned}
& \Sigma_{\mathrm{S} / \mathrm{D}}^{\text {in }}(E)=\Gamma_{\mathrm{S} / \mathrm{D}}(E) f\left(E-E_{\mathrm{S} / \mathrm{D}}^{F}\right) \\
& \Sigma_{\mathrm{S} / \mathrm{D}}^{\text {out }}(E)=\Gamma_{\mathrm{S} / \mathrm{D}}(E)\left[1-f\left(E-E_{\mathrm{S} / \mathrm{D}}^{F}\right)\right]
\end{aligned}
$$

where $f(E)$ is the Fermi distribution, and $E_{\mathrm{S} / \mathrm{D}}^{F}$ denotes the source and drain Fermi energies, respectively. The in/outscattering functions for e-ph interaction are discussed later in Section II-D. The electron and hole correlation functions are then given by

$$
\begin{aligned}
& G^{n}(E)=G \Sigma^{\text {in }} G^{\dagger} \\
& G^{p}(E)=G \Sigma^{\text {out }} G^{\dagger}
\end{aligned}
$$

where the energy dependence of the Green's function and the in/out-scattering functions is suppressed for clarity. The spectral function is [37]

$$
A(E) \equiv i\left(G(E)-G^{\dagger}(E)\right)=G^{n}(E)+G^{p}(E) .
$$

Note that the electron and hole correlation functions $G_{i, j}^{n / p}(E, m)$ are the matrices defined in the basis set of ring numbers $i, j$ and subbands $m$ (we will imply the last index in the rest of this paper). Thus, the diagonal elements $G_{j, j}^{n / p}(E, m)$ correspond to the energy density of carrier occupation at those basis sites (single carbon ring, A or B, in a specific subband) with a given energy $E$. Therefore, the total electron/hole density (per unit length) at a site $z_{j}$ is given by

$$
\begin{aligned}
& n\left(z_{j}\right)=\sum_{m, s} \frac{1}{\Delta z} \int_{-\infty}^{+\infty} \frac{G_{j, j}^{n}(E, m)}{2 \pi} d E \\
& p\left(z_{j}\right)=\sum_{m, s} \frac{1}{\Delta z} \int_{-\infty}^{+\infty} \frac{G_{j, j}^{p}(E, m)}{2 \pi} d E
\end{aligned}
$$

where summation is performed over the spin and subband variables, and it produces the degeneracy factor of 4 (for each nonequivalent subband). In the view of (13), one recognizes 
that the spectral function is proportional to the density of states which is traditionally defined [53] to include the spin summation but is separately taken for each subband

$$
g_{1 \mathrm{D}}\left(E, z_{j}\right)=\frac{A_{j, j}(E, m)}{\pi \Delta z} .
$$

Finally, the current flow from site $z_{j}$ to $z_{j+1}$ in the nearest neighbor tight-binding scheme can be determined from [38] and [39]

$$
\begin{aligned}
I_{j \rightarrow j+1}=\sum_{m, s} \frac{i e}{\hbar} \int_{-\infty}^{+\infty} \frac{d E}{2 \pi} & {\left[H_{j, j+1}(m) G_{j+1, j}^{n}(E, m)\right.} \\
& \left.-H_{j+1, j}(m) G_{j, j+1}^{n}(E, m)\right]
\end{aligned}
$$

wherein the nondiagonal terms of the Hamiltonian (5) contain only the contributions of hopping. The aforementioned equation is a general relationship, in that it is valid even under dissipative transport. Under ballistic conditions, however, (17) further simplifies (for each nonequivalent subband) to

$$
I=\frac{4 e}{\hbar} \int_{-\infty}^{+\infty} \frac{d E}{2 \pi} T(E)\left[f\left(E-E_{S}^{F}\right)-f\left(E-E_{D}^{F}\right)\right]
$$

with the transmission coefficient $T(E)$ given by

$$
T(E)=\operatorname{Trace}\left[\Gamma_{S}(E) G(E) \Gamma_{D}(E) G^{\dagger}(E)\right] .
$$

Equation (19) is the famous Landauer equation that is widely used in mesoscopic transport [37].

One can better understand the bandstructure of CNTs by solving for the eigenvalues of the Hamiltonian (5) for zero external potential and, thereby, obtaining [48] the energy dispersion relations $E\left(k_{z}\right)$ versus the momentum along the length of the tube for each subband. For the lowest conduction and the highest valence subbands close to the $K$-points, the graphene band edge is approximately conic, thus

$$
\left(\frac{2 E}{E_{g}}\right)^{2}=1+\left(\frac{k_{z}}{\Delta k}\right)^{2}
$$

with the bandgap

$$
E_{g}=2 \nu_{F} \hbar \Delta k
$$

and the distance to the $K$-point of

$$
\Delta k=\frac{2}{3 d_{t}} .
$$

The velocity of carriers in the band is

$$
\nu=\frac{d E}{\hbar d k_{z}} .
$$

Far enough from the band edge, the velocity tends to the constant value

$$
\nu_{F}=\frac{3 a_{\mathrm{cc}} t}{2 \hbar} \approx 10^{6} \mathrm{~m} / \mathrm{s} .
$$

The 1-D density of states, including spin summation but only one subband (valley), can thus be expressed as

$$
g_{1 \mathrm{D}}(E)=\frac{2}{\pi \hbar \nu(E)}
$$

or, in other terms

$$
g_{1 \mathrm{D}}(E)=\frac{2}{\pi \hbar \nu_{F}} \cdot \frac{|E|}{\sqrt{E^{2}-\left(E_{g} / 2\right)^{2}}} .
$$

\section{B. Poisson's Equation}

This section summarizes the implementation of selfconsistent electrostatics in our simulation. The diagonal entries of the Hamiltonian in (5) contain the electrostatic potential on the tube surface, which thereby enters the NEGF calculation of charge distribution in (14) and (15). On the other hand, the electrostatic potential and the charge distribution are coupled through the Poisson's equation as well, leading to the Poisson-NEGF self-consistency requirement. The 2-D Poisson equation for the cylindrical transistor geometry in Fig. 1(a) is

$$
\nabla^{2} U(r, z)=-\frac{\rho(r, z)}{\varepsilon} .
$$

Here, $\rho(r, z)$ is the net charge density distribution which includes dopant density as well. At this point, it should be noted that, even though (14) and (15) give the total carrier densities distributed throughout the whole energy range, what we really need in determining the self-consistent potential on the tube surface $U_{j} \equiv U\left(r=r_{\mathrm{CNT}}, z_{j}\right)$ is the induced charge density $\left(r_{\mathrm{CNT}}=\mathrm{CNT}\right.$ radius $)$. This can be determined by performing the integrals in (14) and (15) in a limited energy range defined with respect to the local charge neutrality energy $E_{N}$ [48], [54]. In a semiconducting CNT, due to the symmetry of the conduction and valence bands, $E_{N}$ is expected to be at the midgap energy. Finally, the induced charge density at site $z_{j}$ can be calculated from [48]

$Q_{\text {ind }}\left(z_{j}\right)=\frac{4}{\Delta z}$

$\times\left[(-e) \int_{E_{N}(j)}^{+\infty} \frac{G_{j, j}^{n}(E)}{2 \pi} d E+(+e) \int_{-\infty}^{E_{N}(j)} \frac{G_{j, j}^{p}(E)}{2 \pi} d E\right]$

where the first and second terms correspond to the induced electron and hole densities, respectively, with charge of the electron $e$.

Knowing the induced charge $Q_{\text {ind }}$, the net charge distribution $\rho(r, z)$ is given by

$$
\begin{aligned}
\rho\left(r=r_{\mathrm{CNT}}, z_{j}\right) & =Q_{\text {ind }}\left(z_{j}\right)+N_{D}^{+}-N_{A}^{-} \\
\rho\left(r \neq r_{\mathrm{CNT}}, z\right) & =0
\end{aligned}
$$

where $N_{D}^{+}$and $N_{A}^{-}$are the ionized donor and acceptor concentrations, respectively. Here, it is assumed that the induced charge and the dopants are uniformly distributed over the CNT surface. Finally, (27) is solved to determine the self-consistent 
electrostatic potential $U_{j}$ along the tube surface. The finite difference solution scheme for the 2-D Poisson equation is described in [48]. The calculated potential $U_{j}^{\text {new }}$ gives rise to a modified Hamiltonian (5), eventually leading to the selfconsistent loop between the electrostatics and the quantum transport.

Even though the self-consistent procedure that we have just outlined appears conceptually straightforward, it has poor convergence properties. Therefore, a nonlinear treatment of the Poisson solution is used in practice, as explained in [38] and [55], in order to expedite the electrostatic convergence. The convergence criterion is used in this process to monitor the maximum change in the potential profile between consecutive iterations, i.e., $\max \left(\left|U_{j}^{\text {old }}-U_{j}^{\text {new }}\right|\right) \leq U^{\text {tol }}$, where the tolerance value $U^{\text {tol }}$ is normally taken to be $1 \mathrm{meV}$.

\section{Phonon Modes}

The parameters of the phonons are obviously determined by the structure of the nanotube lattice. The 1-D mass density of an $(n, 0)$ nanotube is

$$
\rho_{1 \mathrm{D}}=\frac{m_{C} n}{\Delta z}
$$

where $m_{C}$ is the mass of a carbon atom. The energy of a phonon of momentum $q$ (in the unconfined dimension) is $\hbar \omega_{q}$. The index of the phonon subband $l$ is implicitly combined with the momentum index here. The half amplitude of vibration for one phonon in a tube of length $L$ is [53]

$$
a_{q}=\sqrt{\frac{\hbar}{2 \rho_{1 \mathrm{D}} L \omega_{q}}} .
$$

For the reservoir in a thermal equilibrium at temperature $T$, the occupation of modes is given by the Bose-Einstein distribution

$$
n_{q}=\left(\exp \left(\frac{\hbar \omega_{q}}{k_{B} T}\right)-1\right)^{-1}
$$

As discussed earlier, the electron states in semiconducting CNTs have a twofold valley degeneracy with the lowest energy subbands having angular quantum numbers $m_{L 1}$ and $m_{L 2}$. The e-ph scattering is governed by energy and momentum conservation rules. Thus, as shown in Fig. 2(a), electrons can be scattered within the same subband (intravalley), where they do not change their angular momentum, and such scattering is facilitated by zone-center phonons having zero angular momentum $(l=0)$. As shown in Fig. 2(b), it is also possible to have an intervalley scattering mediated by zone-boundary phonons having angular quantum number $l=\left|m_{L 1}-m_{L 2}\right|$. There can also be scattering to higher energy subbands assisted by phonon modes with $l \neq 0$ and $l \neq\left|m_{L 1}-m_{L 2}\right|$ [14], [18]; however, we do not discuss results for such processes in this paper.

We have performed phonon dispersion calculations using the force-constant methods described in [21] and [56]. As a result of this analysis, the matrix element for the e-ph interaction is expressed via the deformation potential $J_{1}=6 \mathrm{eV} / \AA$ and the (a)

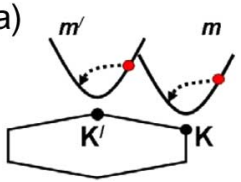

(b)

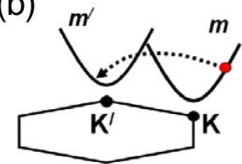

Fig. 2. Lowest energy degenerate subbands in a CNT corresponding to $K$ and $K^{\prime}$ valleys of 2-D graphene Brillouin zone. (a) and (b) show intra- and intervalley scattering processes, respectively.
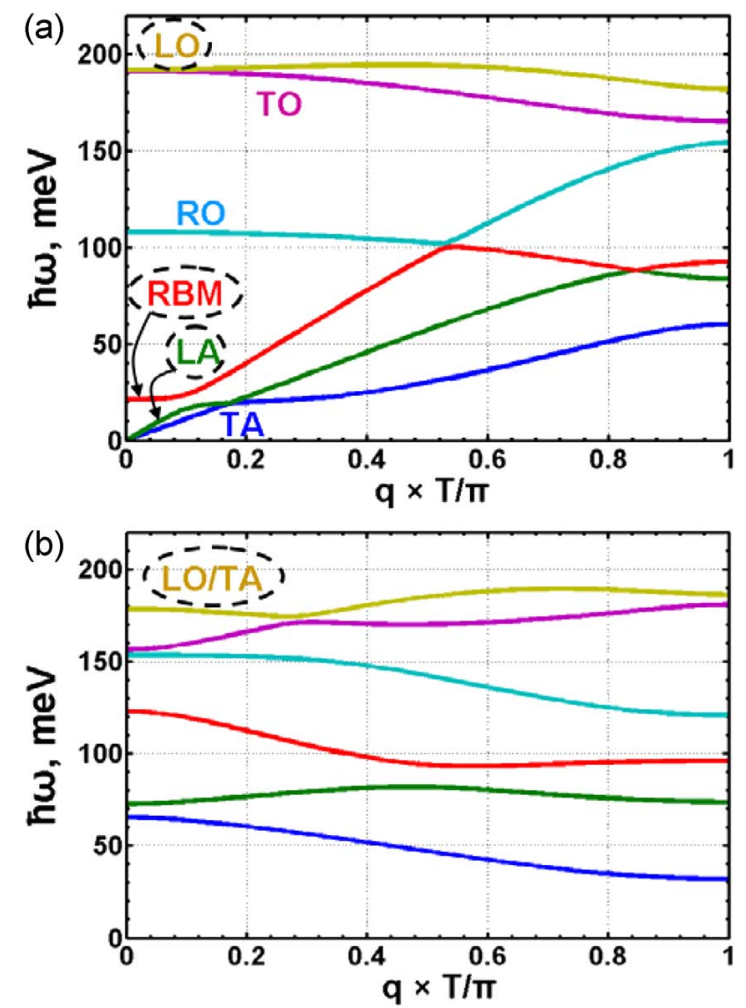

Fig. 3. Energy dispersion for phonon modes in a $(16,0)$ CNT. (a) Zonecenter phonons that allow intravalley scattering. (b) Zone-boundary phonons that allow intervalley scattering. Modes that effectively couple to the electrons are indicated by dashed circles. Zone-boundary phonons are composed of a mixture of fundamental polarizations.

dimensionless matrix element $\left|M_{q}\right|$ as follows: $\left|K_{q}\right|=J_{1}\left|M_{q}\right|$. Zone-center and zone-boundary phonon dispersions for a $(16,0)$ zigzag CNT are shown in Fig. 3(a) and (b), respectively. It is shown that the representation of phonon modes according to fundamental polarizations, such as longitudinal $(L)$, transverse $(T)$, and radial $(R)$, can only be done for the zone-center modes, as indicated in Fig. 3(a). On the other hand, the zoneboundary modes tend to be comprised of a mixture of such fundamental polarizations, as the $\sim 180$-meV mode highlighted in Fig. 3(b), which is mainly a combination of longitudinal optical (LO) and transverse acoustic (TA) polarizations. It should also be noted that the frequency of the radial breathing mode (RBM) calculated here is in very good agreement with the relationship derived from $a b$ initio calculations

$$
\hbar \omega_{\mathrm{RBM}} \approx 28 \mathrm{meV} / d_{t}
$$

where $d_{t}$ is the CNT diameter in nanometers [24], [25], [30]. 
The Hamiltonian of e-ph interaction in a general form is [53]

$$
V=\sum_{q} K_{q} a_{q}\left(b_{q} e^{-i \omega_{q} t+i q r}+b_{q}^{\dagger} e^{i \omega_{q} t-i q r}\right)
$$

where $b_{q}^{\dagger}$ and $b_{q}$ are the creation and annihilation operators for phonons in the mode $q$. The summation over momenta is generally defined via an integral over the first Brillouin zone

$$
\sum_{q}=\left(\frac{L}{2 \pi}\right)^{D} \int d^{D} q
$$

where $D$ is the number of unconfined dimensions. For CNTs, $D=1$, and the limits of the integral are $\pm \pi /\left(3 a_{\mathrm{cc}}\right)$, as follows from (3).

The e-ph coupling calculations have also been carried out, as described in [27], in conjunction with the dispersion calculations in order to account for the mode polarization effect on the e-ph coupling value [56]. We find that only a few phonon modes effectively couple to the electrons. As highlighted in Fig. 3(a), out of the zone-center modes, only the LO (190 meV), LA, and RBM have sufficient coupling, whereas, from zoneboundary modes, only the $180-\mathrm{meV}$ LO/TA mode has significant coupling. Even though we have shown phonon dispersions for a large section of the 1-D Brillouin zone, only the ones close to the zone center (i.e., $q \approx 0$ ) are involved in electron transport [16]. Within that region of the Brillouin zone, all the optical modes are found to have constant energy dispersion, while the acoustic mode has a linear dispersion. Thus, in this paper, all the relevant optical modes for electron transport are considered dispersionless with constant energy $\hbar \omega_{\mathrm{OP}}$, and the zone-center LA mode is taken to be linear with $\omega_{\mathrm{AP}}=\nu_{a} q$ relationship, where $\nu_{a}$ is the sound velocity of that mode. The matrix element of interaction for acoustic phonons (APs) is approximated by a linear function $\left|K_{q}\right|=\tilde{K}_{a}(l) q$. In this paper, we take the matrix elements as inputs and describe the general method of treatment of the e-ph interaction in nanotubes for both the optical (OP) and AP modes.

\section{Electron-Phonon Scattering}

As derived in the Appendix, the in/out-scattering functions for e-ph scattering in a ring $j$ from subband $m^{\prime}$ to subband $m$ are

$$
\begin{aligned}
\sum_{\mathrm{scat}}^{\text {in }}(j, j, m, E)= & D_{0}\left(n_{\omega}+1\right) G^{n}\left(j, j, m^{\prime}, E+\hbar \omega\right) \\
& +D_{0} n_{\omega} G^{n}\left(j, j, m^{\prime}, E-\hbar \omega\right) \\
\Sigma_{\text {scat }}^{\text {out }}(j, j, m, E)= & D_{0}\left(n_{\omega}+1\right) G^{p}\left(j, j, m^{\prime}, E-\hbar \omega\right) \\
& +D_{0} n_{\omega} G^{p}\left(j, j, m^{\prime}, E+\hbar \omega\right) .
\end{aligned}
$$

The imaginary part of self-energy is

$$
\Sigma_{\text {scat }}^{i}(E)=-\frac{i}{2} \Gamma_{\text {scat }}(E)=-\frac{i}{2}\left[\Sigma_{\text {scat }}^{\text {in }}(E)+\Sigma_{\text {scat }}^{\text {out }}(E)\right] .
$$

The real part of self-energy is manifested as a shift of energy levels and is computed by using the Hilbert transform [37]

$$
\Sigma_{\text {scat }}^{r}=P \int \frac{d E^{\prime}}{2 \pi} \frac{\Gamma_{\text {scat }}\left(E^{\prime}\right)}{E-E^{\prime}} .
$$

In this paper, we neglect the real part of e-ph self-energy in order to simplify the computations and because the estimates suggest small influence of the real part. For elastic scattering, i.e., in case it is possible to neglect the energy of a phonon, the in/out-scattering functions are

$$
\begin{aligned}
& \Sigma_{\text {scat }}^{\text {in }}(j, j, m, E)=D_{\mathrm{el}} G^{n}\left(j, j, m^{\prime}, E\right) \\
& \Sigma_{\text {scat }}^{\text {out }}(j, j, m, E)=D_{\mathrm{el}} G^{p}\left(j, j, m^{\prime}, E\right) .
\end{aligned}
$$

In this case, there is no need to neglect the real part of selfenergy, and its complete expression is

$$
\Sigma_{\text {scat }}(j, j, m, E)=D_{\text {el }} G\left(j, j, m^{\prime}, E\right) .
$$

For the OP scattering, the coupling constant is (see the Appendix)

$$
D_{0}=\frac{\hbar\left|K_{0}\right|^{2}}{2 \rho_{1 \mathrm{D}} \omega_{0} \Delta z}
$$

For the AP scattering, the coupling constant is

$$
D_{\mathrm{el}}=\frac{\tilde{K}_{a}^{2} k_{B} T}{\rho_{1 \mathrm{D}} \nu_{a}^{2} \Delta z} .
$$

In the Appendix, we provide the justification in using only the diagonal terms of the self-energy and in/out-scattering functions. We have also made the connection between the in/outscattering functions in the coordinate space and the traditionally considered scattering rates in the momentum space (see [57, Appendix C]).

\section{Numerical Treatment of Dissipative Transport}

Here, we summarize the overall simulation procedure used in this paper. Throughout this paper, we encounter many energy integrals such as (17) and (28). The use of a uniform energy grid becomes prohibitive when sharp features such as quantized energy states need to be accurately resolved. Therefore, an adaptive technique for energy integrations is used based on the quad.m subroutine of Matlab programming language (for the algorithm, see [58]). The treatment of phonon scattering is performed using the self-consistent Born approximation [38], [39]. In that, we need to treat the interdependence of the device Green's function (1) and the scattering self-energy (2) selfconsistently. The treatment of the OP scattering is presented first, followed by that for the AP scattering.

\section{A. Treatment of OP Scattering}

The determination of in/out-scattering functions (37) and (38) for the OP scattering requires the knowledge of the 
TABLE I

Phonon Energy and e-ph Coupling PARAMEters For the CNTs Used In This PAPER

\begin{tabular}{|c|c|c|c|}
\hline Phonon mode & $\begin{array}{c}(16,0) \\
d=1.25 \mathrm{~nm}, \mathrm{E}_{\mathrm{G}}=0.67 \mathrm{eV}\end{array}$ & $\begin{array}{c}(19,0) \\
d=1.50 \mathrm{~nm}, E_{G}=0.56 \mathrm{eV}\end{array}$ & $\begin{array}{c}(22,0) \\
d=1.70 \mathrm{~nm}, E_{G}=0.49 \mathrm{eV}\end{array}$ \\
\hline Intra LO $(190 \mathrm{meV})^{\mathrm{a}}$ & $9.80 \times 10^{-3} \mathrm{eV}^{2}$ & $8.19 \times 10^{-3} \mathrm{eV}^{2}$ & $7.00 \times 10^{-3} \mathrm{eV}^{2}$ \\
\hline Intra $\mathbf{R B M}^{\mathrm{a}, \mathrm{b}}$ & $0.54 \times 10^{-3} \mathrm{eV}^{2}(21 \mathrm{meV})$ & $0.36 \times 10^{-3} \mathrm{eV}^{2}(18 \mathrm{meV})$ & $0.25 \times 10^{-3} \mathrm{eV}^{2}(16 \mathrm{meV})$ \\
\hline Inter LO/TA $(180 \mathrm{meV})^{\mathrm{a}}$ & $19.30 \times 10^{-3} \mathrm{eV}^{2}$ & $16.26 \times 10^{-3} \mathrm{eV}^{2}$ & $14.13 \times 10^{-3} \mathrm{eV}^{2}$ \\
\hline Intra $\mathbf{L A}^{\mathrm{c}}$ & $2.38 \times 10^{-3} \mathrm{eV}^{2}$ & $2.00 \times 10^{-3} \mathrm{eV}^{2}$ & $1.73 \times 10^{-3} \mathrm{eV}^{2}$ \\
\hline
\end{tabular}

a) e-ph coupling for optical phonons is determined according to (44).

b) RBM energy is diameter dependent, and shown in the parentheses.

c) e-ph coupling for acoustic phonons is determined according to (45).

electron and hole correlation functions, specifically, the energyresolved diagonal elements of these functions $G_{j, j}^{n / p}(E)$. It should be noted that only the diagonal elements are needed since we take the scattering self-energy functions to be diagonal in the local interaction approximation [38], [39]. With that, we use the following procedure to determine $G(E)$ and $\Sigma_{\text {scat }}(E)$ self-consistently.

1) Start with the known energy-resolved $G_{j, j}^{n / p}$ distributions. Ballistic distributions are used as the starting point.

2) Determine $\Sigma_{\text {scat }}^{\text {in }}(E), \quad \Sigma_{\text {scat }}^{\text {out }}(E)$, and $\Sigma_{\text {scat }}(E)$ using (37)-(39), respectively, at a given energy $E$.

$3)$ Determine new $G(E)$ using (1).

4) Now, determine new $G^{n}(E)$ and $G^{p}(E)$ from (11) and (12), respectively.

5) Repeat steps 2) through 4) for all energies, and build new $G_{j, j}^{n / p}$ distributions.

6) Repeat steps 1) through 5) until the convergence criterion is satisfied. We use the convergence of the induced carrier density (28) as the criterion.

In the aforementioned calculations, there is a repetitive need for the inversion of a large matrix (1), which can be a computationally expensive task. However, we only need a few diagonals of the eventual solution, such as the main diagonal of $G^{n / p}$ for the calculation of scattering and carrier densities, and the upper/lower diagonals of $G^{n}$ for the calculation of current in (17). The determination of these specific diagonals, in the nearest neighbor tight-binding scheme, can be performed using the efficient algorithms given in [59]. A Matlab implementation of these algorithms can be found in [60]. Finally, it should be noted that the overall accuracy of the Born convergence procedure previously described is confirmed at the end by observing the current continuity throughout the device (17).

\section{B. Treatment of AP Scattering}

Similar to the aforementioned method, the AP scattering is treated using the following procedure.

1) Start with the known energy-resolved $G_{j, j}^{n / p}$ distributions. Ballistic distributions are used as the starting point.
2) Determine $\Sigma_{\text {scat }}^{\text {in }}(E), \Sigma_{\text {scat }}^{\text {out }}(E)$, and $\Sigma_{\text {scat }}(E)$ using (41)-(43), respectively, at a given energy $E$.

$3)$ Determine new $G(E)$ using (1).

4) Now, determine new $G^{n}(E)$ and $G^{p}(E)$ at energy $E$ from (11) and (12), respectively.

5) Repeat steps 2) through 4) until the convergence criterion is satisfied. Here, we use the convergence of $G^{n}(E)$.

6) Repeat steps 2) through 5) for all energies, and build new $G_{j, j}^{n / p}$ distributions.

7) Repeat steps 1) through 6) until the convergence criterion is satisfied. We use the convergence of the induced carrier density (28) as the criterion.

For the case of AP scattering, we have introduced an additional convergence loop (step 5) in the list) since, unlike in inelastic scattering, here the self-consistent Born calculation at a given energy is decoupled from that at all other energy values. Similar to the OP scattering, we use the efficient algorithms of [59] for numerical calculations and confirm the overall accuracy of the convergence procedure by monitoring current continuity throughout the device.

\section{RESUlTs AND Discussion}

Dissipative transport simulations are carried out, as explained in the previous sections, and the results are compared to that with the ballistic transport. Here, we first study the effects of phonon scattering on the CNTFET characteristics using a $(16,0)$ tube as a representative case. Then, we compare the diameter dependence using $(16,0),(19,0)$ and $(22,0)$ tubes that belong to the $\bmod (n-m, 3)=1$ family. The device parameters [Fig. 1(a)] used for the simulation of OP scattering are as follows: $L_{\mathrm{ch}}=20 \mathrm{~nm}, L_{\mathrm{SD}}=30 \mathrm{~nm}, L_{\mathrm{ext}}=0, t_{\mathrm{OX}}=$ $2 \mathrm{~nm}\left(\mathrm{HfO}_{2}\right.$ with $\left.\kappa=16\right)$, and the source/drain doping $N_{\mathrm{SD}}=$ $1.5 / \mathrm{nm}$. This doping concentration should be compared with the carbon atom density of $\left(4 n / 3 a_{\mathrm{cc}}\right)$ in an $(n, 0)$ zigzag CNT, which is $\sim 150 / \mathrm{nm}$ in a $(16,0)$ tube. For the simulation of AP scattering, a heavy-doped extension region is used for better convergence of the electrostatic solution. In this case, $L_{\mathrm{SD}}=$ $20 \mathrm{~nm}, L_{\mathrm{ext}}=15 \mathrm{~nm}, N_{\mathrm{SD}}=1.5 / \mathrm{nm}$, and the extension doping $N_{\text {ext }}=1.8 / \mathrm{nm}$ are used, and all the other parameters are the same as for the previous case. Except for assisting 


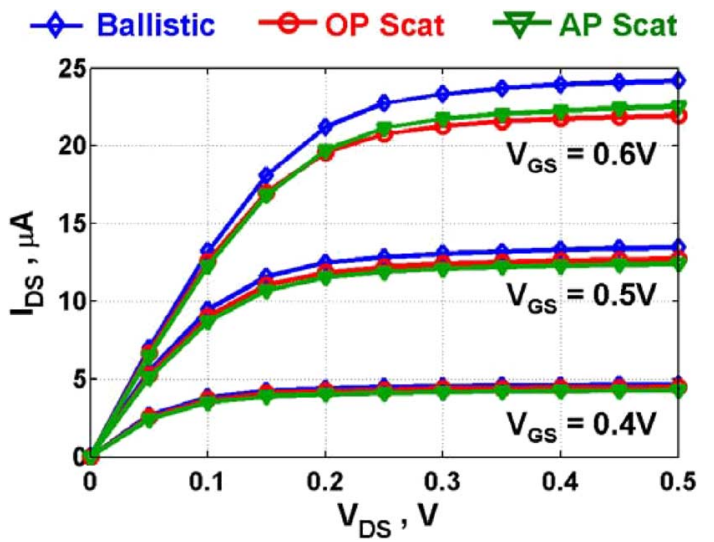

Fig. 4. $I_{\mathrm{DS}}-V_{\mathrm{DS}}$ for the $(16,0)$ CNTFET under ballistic transport, OP scattering (all modes together), and AP scattering. High-energy OP scattering becomes important at sufficiently large gate biases. Until then, AP and RBM scattering are dominant.

in the convergence procedure, the effect of the heavy-doped extensions on the device characteristics is negligible. It should be noted that, under the OP scattering, we consider the impact of intra-LO, intra-RBM, and inter-LO/TA phonon modes all together simultaneously (Table I). The intra-LA mode is treated under the AP scattering separately.

Fig. 4 compares the $I_{\mathrm{DS}}-V_{\mathrm{DS}}$ results for the $(16,0)$ CNTFET under ballistic transport and that with the OP and AP scattering. It is shown that phonon scattering can indeed have an appreciable effect on the device ON-current: At $V_{\mathrm{GS}}=0.6 \mathrm{~V}$, the ON-current is reduced by $\sim 9 \%$ and $\sim 7 \%$ due to the OP and AP scattering, respectively. The relative importance of the two scattering mechanisms also shows an interesting behavior. Up to moderate gate biases, the effect of AP scattering is stronger $\left(V_{\mathrm{GS}} \leq 0.5 \mathrm{~V}\right)$. At large gate biases, the OP scattering becomes the more important process $\left(V_{\mathrm{GS}} \geq 0.6 \mathrm{~V}\right)$. This relative behavior can be better observed in the $I_{\mathrm{DS}}-V_{\mathrm{GS}}$ results shown in Fig. 5. Here, it is shown that, up to moderate gate biases, the AP scattering causes a larger reduction in the device current compared to the OP scattering. Furthermore, the current reduction shown in this case for the OP scattering is mainly due to the low-energy RBM mode [32]. At large gate biases, however, the effect of OP scattering becomes stronger, reducing the current by $\sim 16 \%$ from the ballistic level at $V_{\mathrm{GS}}=0.7 \mathrm{~V}$. Previous studies have shown that the strong current degradation at larger gate biases is due to high-energy OP scattering processes becoming effective (mainly, the inter-LO/TA and intra-LO modes) [31], [32]. Nevertheless, the importance of AP and low-energy RBM scattering should be appreciated since these might be the relevant scattering mechanisms under typical biasing conditions of a nanoscale transistor [61].

The relative behavior of OP and AP scattering can be understood by studying Fig. 6. It shows the energy-positionresolved current spectrum, which is essentially the integrand of (17), under ballistic transport and OP scattering. In Fig. 6(a), it is shown that, under ballistic conditions, carriers injected from the source reach the drain without losing energy inside the device region. There exists a finite density of current below the conduction band edge $\left(E_{C}\right)$ which is due to the

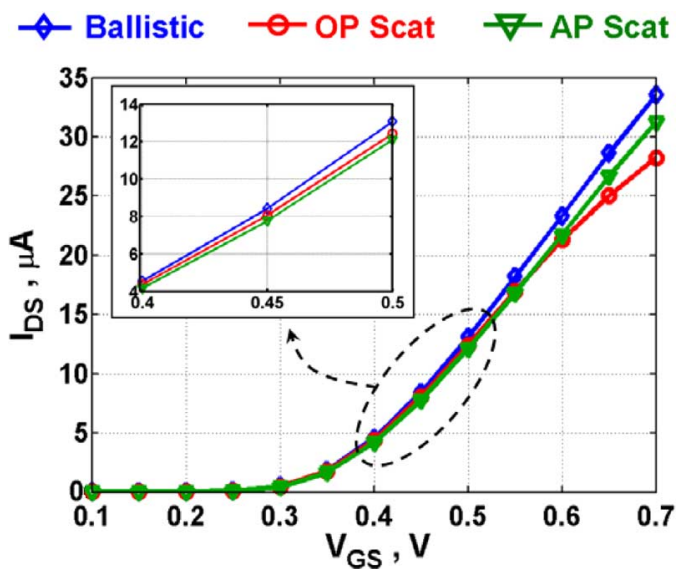

Fig. 5. $\quad I_{\mathrm{DS}}-V_{\mathrm{GS}}$ for the $(16,0) \mathrm{CNTFET}$ at $V_{\mathrm{DS}}=0.3 \mathrm{~V}$ under ballistic transport, OP scattering (all modes together), and AP scattering. The inset shows that APs are more detrimental up to moderate gate biases.
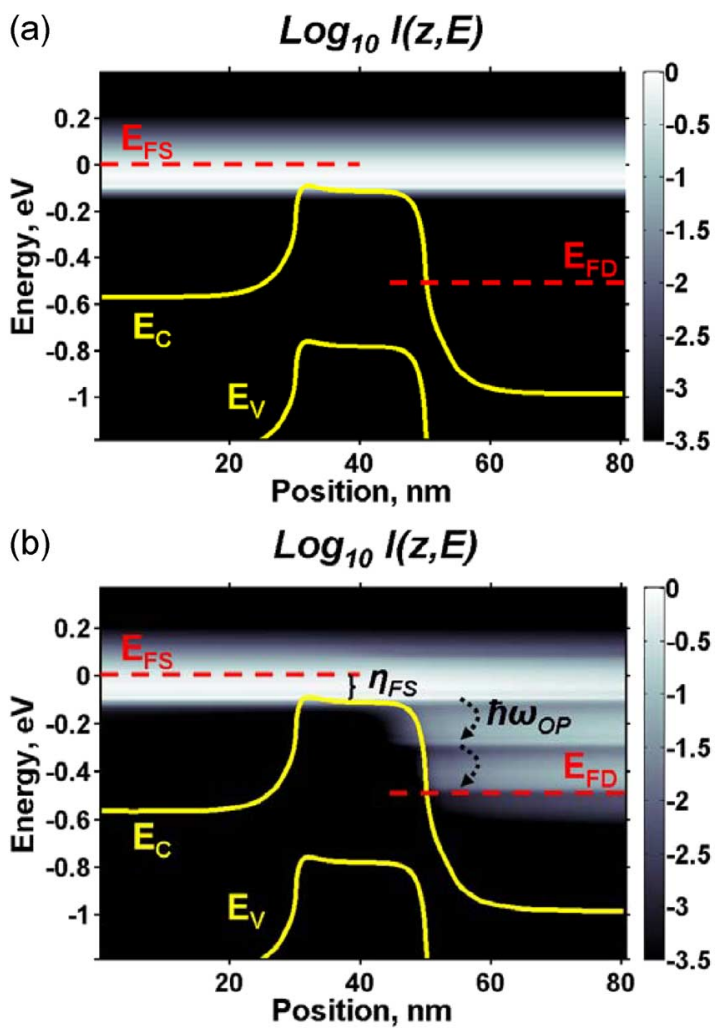

Fig. 6. Energy-position-resolved current spectrum for $(16,0)$ CNTFET at $V_{\mathrm{GS}}=0.5 \mathrm{~V}$ and $V_{\mathrm{DS}}=0.5 \mathrm{~V}$ (logarithmic scale). (a) Ballistic and (b) dissipative transports (all OP modes together). Thermalization near the drain end by emitting high-energy OPs leaves the electrons without enough energy to overcome the channel barrier.

quantum-mechanical tunneling. In the presence of OP scattering, however, it is shown that the carriers near the drain end relax to low energy states by emitting phonons [Fig. 6(b)]. Nevertheless, up to moderate gate biases, high-energy OP scattering does not affect the device current due to the following reason. For such biasing conditions, the energy difference between the source Fermi level and the top of the channel barrier $\eta_{\mathrm{FS}}$ is smaller than the OP energy: $\eta_{\mathrm{FS}} \ll \hbar \omega_{\mathrm{OP}}$. Therefore, a majority of the positive going carriers (source $\rightarrow$ drain) in the channel region does not experience high-energy OP scattering, 

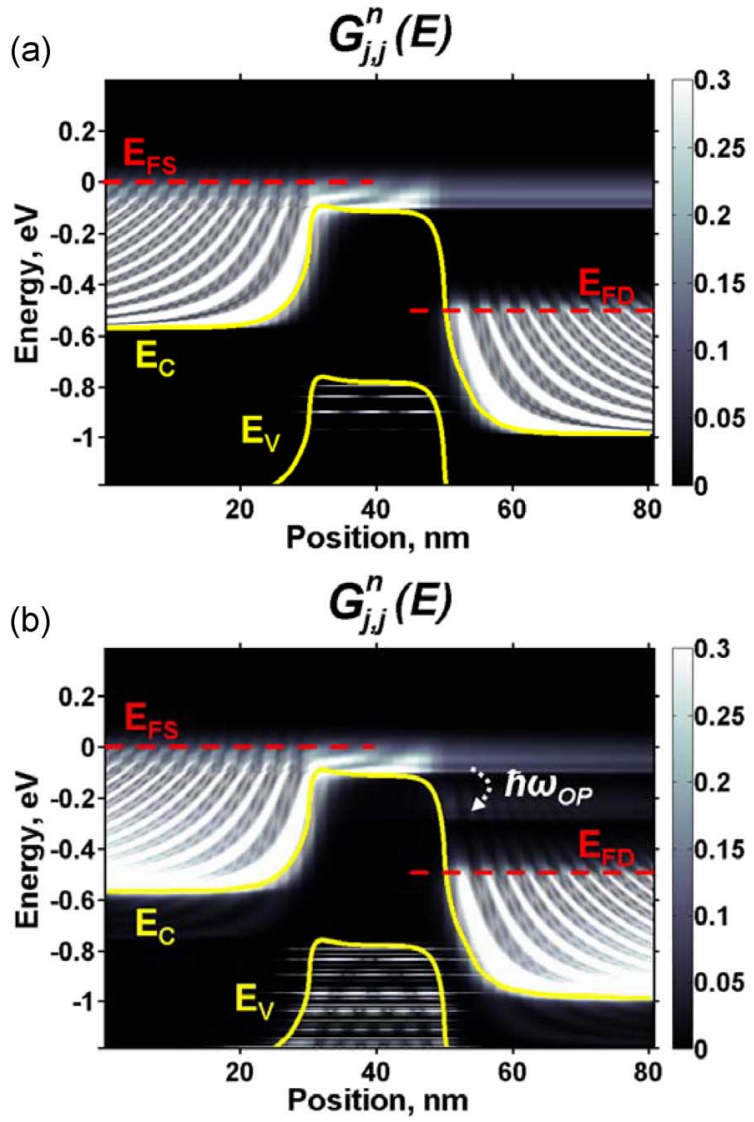

Fig. 7. Energy-position-resolved electron density spectrum for $(16,0)$ CNTFET at $V_{\mathrm{GS}}=0.5 \mathrm{~V}$ and $V_{\mathrm{DS}}=0.5 \mathrm{~V}$. (a) Ballistic and (b) dissipative transports (all OP modes together). Quantized states in the valence band are broadened and give rise to many phonon-induced sidebands. The interference pattern for conduction band states is also broadened compared to the ballistic case.

except for a minute portion in the high-energy tail of the source Fermi distribution. On the other hand, when these carriers reach the drain end, there are empty low-lying states that they scatter to. After emitting a high-energy OP, however, these carriers do not have enough energy to surmount the channel barrier and reach the source region again. Thus, the effect of high-energy OP scattering on the device current is suppressed until backscattering becomes effective at larger gate biases for $\eta_{\mathrm{FS}} \geq \hbar \omega_{\mathrm{OP}}$. On the other hand, the low-energy RBM phonons and APs can effectively backscatter at all gate biases. They are the dominant scattering mechanisms until the high-energy $\mathrm{OP}$ becomes important at large biases [31], [32].

Fig. 7 shows the energy-position-resolved electron density spectrum, which is essentially the integrand of (14). By examining Fig. 7(a), one can see that electrons are filled up to the respective Fermi levels in the two contact regions. In these regions, a characteristic interference pattern in the distribution function is observed due to the quantum-mechanical inference of positive and negative going states [48]. Quantized valence band states in the channel region are due to the longitudinal confinement in this effective potential well [48]. In the presence of OP scattering, a few interesting features are observed in Fig. 7(b). The interference pattern seen in the contact regions is smeared due to the broadening of energy states by
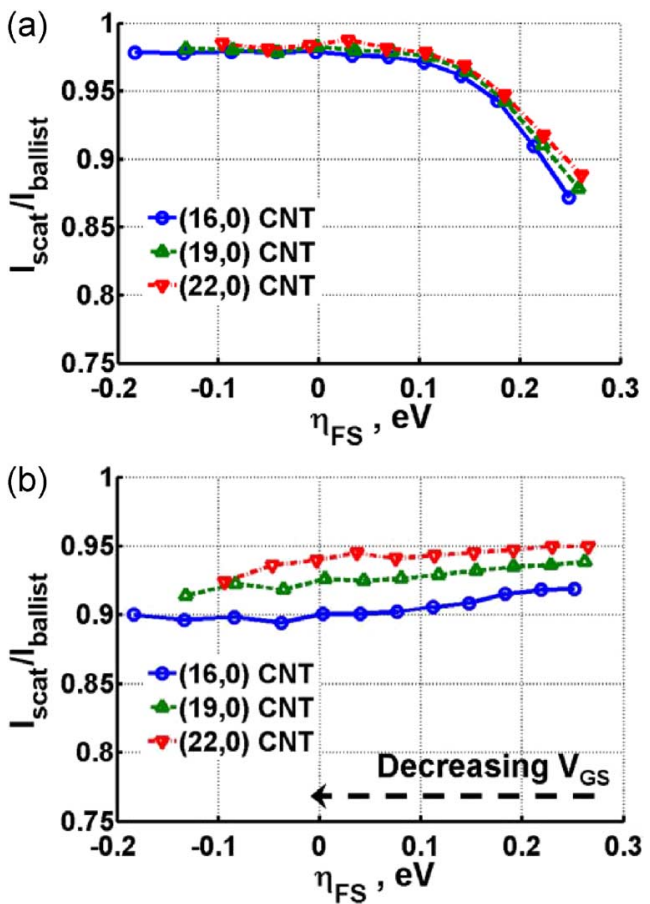

Fig. 8. Ballisticity $\left(I_{\text {scat }} / I_{\text {ballist }}\right)$ versus $\eta_{\mathrm{FS}}$ for $(16,0),(19,0)$ and $(22,0)$ CNTFETs. (a) With all OP modes together. (b) With AP scattering. $\eta_{\mathrm{FS}}$ is defined as the energy difference between the source Fermi level and the channel barrier [see Fig. 6(b)].

incoherent OP scattering. The electrons near the drain end relax down to the low-lying empty states, even though they are less discernible in the linear color scale employed here. More interestingly, now, we observe a multitude of quantized valence band states in the channel region. Such states with energies below the conduction band edge of the drain region are observed here due to their additional broadening by coupling to the phonon bath. They were unobservable in the ballistic case since they lied inside the bandgap regions of the contact reservoirs that led to zero contact broadening $\Gamma_{\mathrm{S} / \mathrm{D}} \approx 0$. The additional low-intensity states observed are the phonon-induced sidebands of the main quantized levels originating from the variety of OP modes considered here. Carrier transport through these quantized states is indeed possible under appropriate biasing conditions and lead to many interesting properties such as less than $60-\mathrm{mV} /$ decade subthreshold operation and phononassisted inelastic tunneling (see [34] and [35]).

Fig. 8 explores the diameter dependence of the impact of phonon scattering in CNTFETs. As mentioned earlier, we consider the $\bmod (n-m, 3)=1$ type of tubes. Similar trends in the behavior can be expected for the $\bmod (n-m, 3)=2$ family as well [28], [29]. Here, we compare the ballisticity of tubes, defined as the ratio between the current under scattering and the ballistic current $\left(I_{\text {scat }} / I_{\text {ballist }}\right)$ versus $\eta_{\mathrm{FS}}$, defined in Fig. 6(b). Positive $\eta_{\mathrm{FS}}$ corresponds to the ON-state of the device at large positive gate biases, and negative $\eta_{\mathrm{FS}}$ is for the OFFstate. The characteristic rolloff of ballisticity under the OP scattering is shown in Fig. 8(a) [32]. In that, the rolloff is due to the high-energy OP scattering which is becoming effective at large gate biases. The ballisticity reduction at small gate biases is due to the low-energy RBM scattering [32]. In Fig. 8(a), 
it is shown that the impact of high-energy OP scattering decreases for larger diameter tubes. This can be easily understood by noting that the e-ph coupling parameter for these modes (intra-LO and inter-LO/TA) monotonically decreases with an increasing diameter (Table I). On the other hand, the impact of the RBM mode at low gate biases seems to be nearly diameter independent for the tubes considered here, even though there is a similar decrease in the e-ph coupling for larger diameter tubes (Table I). This behavior is due to the concomitant reduction of energy of the RBM mode at larger diameters that leads to an increased amount of scattering events, which ultimately cancels out the overall impact on the device current.

Diameter dependence of AP scattering is shown in Fig. 8(b). The ballisticity for larger tubes is higher due to the corresponding reduction of the e-ph coupling parameter shown in Table I. They all show a slight increase in the ballisticity at larger gate biases due to the majority of the positive going carriers occupying states well above the channel conduction band edge [32]. The backscattering rate is a maximum near the band edge due to the increased 1-D density of states and decays at larger energies [14], [16], [18]. It is shown in Fig. 8 that, for all the tubes, the impact of AP scattering is stronger compared to the OP scattering until the high-energy modes become effective. Under typical biasing conditions for nanoscale transistor operation, $\eta_{\mathrm{FS}}$ will be limited $\left(\eta_{\mathrm{FS}} \leq 0.15 \mathrm{eV}\right)$, and the transport will be dominated by the AP and low-energy RBM scattering [61].

\section{CONCLUSION}

In conclusion, we present here the detailed self-contained description of the NEGF method to simulate transport of carriers in the CNT transistors with the account of both quantum effects and e-ph scattering. This capability is particularly necessary, since it provides the rigorous treatment in the practically important limit of intermediate length devices. We outline our numerical procedure for solution of the NEGF equations via convergence of several self-consistent loops. Finally, we display a few of the simulation results obtained by this method, such as the energy spectra of carrier density and current, and the current-voltage characteristics. They enable a researcher to uncover the workings of the quantum phenomena underlying the operation of carbon-nanotube transistors and to predict their performance.

\section{APPENDIX}

\section{DERIVATION OF THE IN/OUT-SCATTERING FUNCTIONS FOR THE e-ph INTERACTION}

Although the self-energy for the e-ph scattering has been discussed multiple times, e.g., [37], considerable confusion still exists about its form and assumptions used in the derivation. One reason may be the fact that, in device simulation, one uses Green's functions and self-energy functions of two coordinate arguments, while the scattering processes are traditionally formulated in the momentum-dependent and coordinateindependent representation. The other reason is that the expression for self-energy looks slightly different for different material systems. Here, we aim to derive the expression for the self-energy in a simple but general form and, then, to specify it for the particular case of 1-D transport in the CNTFETs. Similar calculations have been presented for III-V devices [38] and Sinanowire transistors [44], [45].

The self-consistent Born approximation results in the following in/out-scattering functions for the e-ph interactions [38], [62]

$$
\Sigma^{\text {in }, \text { out }}\left(X_{1}, X_{2}\right)=G^{n, p}\left(X_{1}, X_{2}\right) D^{n, p}\left(X_{1}, X_{2}\right)
$$

where the argument $X=\{r, m, t\}$ incorporates the spatial coordinates in the unconfined dimensions, subband/valley index, and time, respectively. The phonon propagator contains the average over the random variables of the reservoir designated by angle brackets

$$
\begin{aligned}
& D^{n}\left(X_{1}, X_{2}\right)=\left\langle V\left(X_{1}\right) V\left(X_{2}\right)\right\rangle \\
& D^{p}\left(X_{1}, X_{2}\right)=\left\langle V\left(X_{2}\right) V\left(X_{1}\right)\right\rangle .
\end{aligned}
$$

The averages of the following operator products in a reservoir at thermal equilibrium depend on the phonon-occupation numbers (33)

$$
\left\langle b_{q}^{\dagger} b_{q^{\prime}}\right\rangle=\delta_{q q^{\prime}} n_{q}, \quad\left\langle b_{q^{\prime}} b_{q}^{\dagger}\right\rangle=\delta_{q q^{\prime}}\left(n_{q}+1\right)
$$

and all other averages of pair products are zero. On substitution of the e-ph Hamiltonian (35), it results in

$$
\begin{aligned}
D^{n}\left(r_{1}, m_{1}, t_{1}, r_{2},\right. & \left.m_{2}, t_{2}\right) \\
=\sum_{q}\left|K_{q}\right|^{2} a_{q}^{2}\left[\left(n_{q}+1\right) \exp \left(i \omega_{q}\left(t_{2}-t_{1}\right)+i q\left(r_{1}-r_{2}\right)\right)\right. & \\
& \left.+n_{q} \exp \left(i \omega_{q}\left(t_{1}-t_{2}\right)+i q\left(r_{2}-r_{1}\right)\right)\right]
\end{aligned}
$$

and a similar expression for $D^{p}\left(r_{1}, l_{1}, t_{1}, r_{2}, l_{2}, t_{2}\right)$. The selection rules for the electron subbands $m, m^{\prime}$ and phonon subbands $l$ are similar as described in Section II-C. Then, we limit the consideration to stationary situation, i.e., where the functions depend only on the difference of times $t=t_{2}-t_{1}$. The Fourier transform relative to this time interval produces energy-dependent in/out-scattering functions (given here for a specific phonon subband)

$$
\begin{aligned}
\Sigma^{\text {in }}\left(r_{1}, r_{2}, m, E\right)= & D\left(r_{1}, r_{2}, l, E\right)\left(n_{q}+1\right) \\
& \times G^{n}\left(r_{1}, r_{2}, m^{\prime}, E+\hbar \omega_{q}\right) \\
& +D^{*}\left(r_{1}, r_{2}, l, E\right) \\
& \times n_{q} G^{n}\left(r_{1}, r_{2}, m^{\prime}, E-\hbar \omega_{q}\right) \\
\Sigma^{\text {out }}\left(r_{1}, r_{2}, m, E\right)= & D^{*}\left(r_{1}, r_{2}, l, E\right)\left(n_{q}+1\right) \\
& \times G^{p}\left(r_{1}, r_{2}, m^{\prime}, E-\hbar \omega_{q}\right) \\
& +D\left(r_{1}, r_{2}, l, E\right) \\
& \times n_{q} G^{p}\left(r_{1}, r_{2}, m^{\prime}, E+\hbar \omega_{q}\right)
\end{aligned}
$$

where the first term in the expressions corresponds to the emission of a phonon, and the second one corresponds to the 
absorption of a phonon. The e-ph coupling operator contains the sum over the phonon momentum that operates on the factors to the right of it

$$
D\left(r_{1}, r_{2}, l\right)=\sum_{q}\left|K_{q}\right|^{2} a_{q}^{2} \exp (-i q r)
$$

It depends on the difference of the spatial coordinates $r=$ $r_{2}-r_{1}$. The expressions for the in/out-scattering functions drastically simplify in the following two cases.

First, for isotropic scattering with phonons of constant energy $\left(\left|K_{q}\right| \approx\left|K_{0}\right|\right.$ and $\omega_{q} \approx \omega_{0}$, and they are independent of $q$ ). This is approximately fulfilled for the OPs. In this case, the e-ph scattering operator reduces to the calculation of a sum

$$
D\left(r_{1}, r_{2}, l\right)=\frac{\hbar\left|K_{0}\right|^{2}}{2 \rho_{1 \mathrm{D}} \omega_{0}} \int_{-\pi /\left(3 a_{\mathrm{cc}}\right)}^{\pi /\left(3 a_{\mathrm{cc}}\right)} \frac{d q}{2 \pi} \exp (-i q r) .
$$

For the distance of integer multiple of the nanotube period $r=$ $j 3 a_{\mathrm{cc}}$, the integral above

$$
\int_{-\pi /\left(3 a_{\mathrm{cc}}\right)}^{\pi /\left(3 a_{\mathrm{cc}}\right)} \frac{d q}{2 \pi} \exp (-i q r)=\left\{\begin{array}{ll}
1 /\left(3 a_{\mathrm{cc}}\right), & j=0 \\
0, & j \neq 0
\end{array} .\right.
$$

One needs to insert the factor of 4 , for the number of rings in the period, to obtain that the e-ph coupling is a constant factor (44)

$$
D_{0}=\frac{\hbar\left|K_{0}\right|^{2}}{2 \rho_{1 \mathrm{D}} \omega_{0} \Delta z}
$$

and the expression for the in/out-scattering functions (37) and (38). In addition, a very important conclusion is that the selfenergy and the in/out-scattering functions can be treated as diagonal in this case. This significantly simplifies the problem and permits the use of various algorithms of solution of the matrix equations that are only applicable to three-diagonal matrices, such as the recursive inversion method [38].

Second case, for elastic scattering, when one can neglect the energy of a phonon compared to characteristic energy differences. This is approximately fulfilled for the APs. For this case, the dependence on the momentum is typically $\omega_{q}=\nu_{a}(l) q$ and $\left|K_{q}\right|=\tilde{K}_{a}(l) q$, and only phonons with momentum close to $q=0$ have the appreciable occupations, such that

$$
n_{q} \approx \frac{k_{B} T}{\hbar \omega_{q}} \gg 1
$$

Then again, as in (53), the matrix element and the number of phonon factors prove to be independent of the phonon momentum and can be taken out of the summation

$$
\begin{aligned}
\Sigma^{\operatorname{in}}\left(r_{1}, r_{2}, m, E\right) & =G^{n}\left(r_{1}, r_{2}, m^{\prime}, E\right) \\
& \times \int_{-\pi /\left(3 a_{\mathrm{cc}}\right)}^{\pi /\left(3 a_{\mathrm{cc}}\right)} \frac{k_{B} T}{\hbar \omega_{q}} \frac{\hbar \tilde{K}_{a}^{2} q^{2}}{2 \rho_{1 \mathrm{D}} \omega_{q}} \frac{d q}{2 \pi} \exp (-i q r)+\text { c.c. }
\end{aligned}
$$

to again yield a diagonal in/out-scattering functions and (42)

$$
\Sigma^{\mathrm{in}}\left(r_{1}, r_{1}, m, E\right)=2 \frac{k_{B} T \tilde{K}_{a}^{2}}{2 \rho_{1 \mathrm{D}} \nu_{a}^{2}} G^{n}\left(r_{1}, r_{1}, m^{\prime}, E\right) \frac{4}{3 a_{\mathrm{cc}}}
$$

and the constant elastic e-ph coupling (45). Note an additional factor of 2 in these expressions because the processes with emission and absorption of a phonon are now lumped into one term.

By going beyond the assumption of a constant product of the coupling factor and the phonon occupation, we can determine how good the approximation of a diagonal self-energy is. By representing it as a Taylor series (and we know that it is an even function)

$$
\left|K_{q}\right|^{2} a_{q}^{2} n_{q}=\left|K_{0}\right|^{2} a_{0}^{2} n_{0}\left(1+\frac{q^{2}}{q_{(2)}^{2}}+\cdots\right)
$$

and examining the second term, we obtain

$$
\begin{aligned}
\int_{-\pi /\left(3 a_{\mathrm{cc}}\right)}^{\pi /\left(3 a_{\mathrm{cc}}\right)} \frac{d q}{2 \pi} \cdot \frac{q^{2}}{q_{(2)}^{2}} & \exp (-i q r) \\
& =\left\{\begin{array}{ll}
\pi^{2} /\left(q_{(2)}^{2} 3^{4} a_{\mathrm{cc}}^{3}\right), & j=0 \\
2(-1)^{j} /\left(j q_{(2)}^{2} 3^{3} a_{\mathrm{cc}}^{3}\right), & j \neq 0
\end{array} .\right.
\end{aligned}
$$

This can be restated as: The off-diagonal terms of the selfenergy and the in/out-scattering functions have the order of magnitude of the variation of the product (59) over the first Brillouin zone. By doing an inverse Fourier transform of (59), we recognize the parameter $q_{(2)}$ as the inverse characteristic radius of the e-ph interaction. Thus, the alternative formulation of the aforementioned criterion is as follows: The self-energy is diagonal if the corresponding interaction radius is much less than the crystal lattice size.

\section{ACKNOWLEDGMENT}

The authors would like to thank the NSF Network for Computational Nanotechnology for the computational support. S. O. Koswatta would like to thank the Intel Foundation for the Ph.D. Fellowship support.

\section{REFERENCES}

[1] S. J. Tans, A. R. M. Verschueren, and C. Dekker, "Room temperature transistor based on a single carbon nanotube," Nature, vol. 393, no. 6680, p. 49, May 1998.

[2] R. Martel, T. Schmidt, H. R. Shea, T. Hertel, and P. Avouris, "Singleand multi-wall carbon nanotube field-effect transistors," Appl. Phys. Lett., vol. 73, no. 17, pp. 2447-2449, Oct. 1998.

[3] M. P. Anantram and F. Leonard, "Physics of carbon nanotube electronic devices," Rep. Prog. Phys., vol. 69, no. 3, pp. 507-561, Mar. 2006.

[4] S. Frank, P. Poncharal, Z. L. Wang, and W. A. de Heer, "Carbon nanotube quantum resistor," Science, vol. 280, no. 5370, pp. 1744-1746, Jun. 1998.

[5] J. Kong, E. Yenilmez, T. W. Tombler, W. Kim, and H. Dai, "Quantum interference and ballistic transmission in nanotube electron waveguide," Phys. Rev. Lett., vol. 87, no. 10, p. 106801 , Aug. 2001. 
[6] A. Javey, J. Guo, Q. Wang, M. Lundstrom, and H. Dai, "Ballistic carbon nanotube field-effect transistors," Nature, vol. 424, no. 6949, pp. 654-657, Aug. 2003.

[7] A. Javey et al., "Self-aligned ballistic molecular transistor and electrically parallel nanotube arrays," Nano Lett., vol. 4, no. 7, pp. 1319-1322, 2004.

[8] Y.-M. Lin, J. Appenzeller, Z. Chen, Z.-G. Cgen, H.-M. Cheng, and P. Avouris, "High-performance dual-gate carbon nanotube FETs with $40 \mathrm{~nm}$ gate length," IEEE Electron Device Lett., vol. 26, no. 11, pp. 823825, Nov. 2005.

[9] T. Durkop, S. A. Getty, E. Cobas, and M. S. Fuhrer, "Extraordinary mobility in semiconducting carbon nanotubes," Nano Lett., vol. 4, no. 1, pp. 35-39, 2004

[10] X. Zhou, J.-Y. Park, S. Huang, J. Liu, and P. L. McEuen, "Band structure, phonon scattering, and the performance limit of single-walled carbon nanotube transistors," Phys. Rev. Lett., vol. 95, no. 14, p. 146805 , Sep. 2005

[11] Z. Yao, C. L. Kane, and C. Dekker, "High-field electrical transport in single-wall carbon nanotubes," Phys. Rev. Lett., vol. 84, no. 13, pp. 2941-2944, Mar. 2000.

[12] A. Javey, J. Guo, M. Paulsson, Q. Wang, D. Mann, M. Lundstrom, and H. Dai, "High-field quasiballistic transport in short carbon nanotubes," Phys. Rev. Lett., vol. 92, no. 10, p. 106 804, 2004.

[13] J.-Y. Park et al., "Electron-phonon scattering in metallic single-walled carbon nanotubes," Nano Lett., vol. 4, no. 3, pp. 517-520, 2004.

[14] G. Pennington and N. Goldsman, "Semiclassical transport and phonon scattering of electrons in semiconducting carbon nanotubes," Phys. Rev. B, Condens. Matter, vol. 68, no. 4, p. 045 426, Jul. 2003.

[15] G. Pennington and N. Goldsman, "Low-field semiclassical carrier transport in semiconducting carbon nanotubes," Phys. Rev. B, Condens. Matter, vol. 71, no. 20, p. 205318 , May 2005.

[16] V. Perebeinos, J. Tersoff, and P. Avouris, "Electron-phonon interaction and transport in semiconducting carbon nanotubes," Phys. Rev. Lett., vol. 94, no. 8, p. 086802 , Mar. 2005.

[17] V. Perebeinos, J. Tersoff, and P. Avouris, "Mobility in semiconducting carbon nanotubes at finite carrier density," Nano Lett., vol. 6, no. 2, pp. 205-208, 2006.

[18] A. Verma, M. Z. Kauser, and P. P. Ruden, "Ensemble Monte Carlo transport simulations for semiconducting carbon nanotubes," J. Appl. Phys., vol. 97, no. 11, p. 114319 , Jun. 2005

[19] A. Verma, M. Z. Kauser, and P. P. Ruden, "Effects of radial breathing mode phonons on charge transport in semiconducting zigzag carbon nanotubes," Appl. Phys. Lett., vol. 87, no. 12, p. 123 101, Sep. 2005.

[20] H. C. d'Honincthun, S. Galdin-Retailleau, J. See, and P. Dollfus, "Electron-phonon scattering and ballistic behavior in semiconducting carbon nanotubes," Appl. Phys. Lett., vol. 87, no. 17, p. 172112 , Oct. 2005.

[21] R. Saito, G. Dresselhaus, and M. S. Dresselhaus, Physical Property of Carbon Nanotubes. London, U.K.: Imperial College Press, 1998.

[22] H. Suzuura and T. Ando, "Phonons and electron-phonon scattering in carbon nanotubes," Phys. Rev. B, Condens. Matter, vol. 65, no. 23, p. 235412 , May 2002.

[23] S. V. Goupalov, "Continuum model for long-wavelength phonons in twodimensional graphite and carbon nanotubes," Phys. Rev. B, Condens. Matter, vol. 71, no. 8, p. 085 420, Feb. 2005.

[24] J. Kurti, G. Kresse, and H. Kuzmany, "First-principles calculation of the radial breathing mode of single-wall carbon nanotubes," Phys. Rev. B, Condens. Matter, vol. 58, no. 14, pp. R8869-R8872, Oct. 1998.

[25] D. Sanchez-Portal, E. Artacho, J. M. Soler, A. Rubio, and P. Ordejon, "Ab initio structural, elastic, and vibrational properties of carbon nanotubes," Phys. Rev. B, Condens. Matter, vol. 59, no. 19, pp. 12678-12 688, May 1999.

[26] O. Dubay and G. Kresse, "Accurate density functional calculations for the phonon dispersion relations of graphite layer and carbon nanotubes," Phys. Rev. B, Condens. Matter, vol. 67, no. 3, p. 035 401, Jan. 2003.

[27] G. D. Mahan, "Electron-optical phonon interaction in carbon nanotubes," Phys. Rev. B, Condens. Matter, vol. 68, no. 12, p. 125 409, Sep. 2003.

[28] J. Jiang, R. Saito, G. G. Samsonidze, S. G. Chou, A. Jorio, G. Dresselhaus, and M. S. Dresselhaus, "Electron-phonon matrix elements in singlewall carbon nanotubes," Phys. Rev. B, Condens. Matter, vol. 72, no. 23, p. $235408,2005$.

[29] V. N. Popov and P. Lambin, "Intraband electron-phonon scattering in single-walled carbon nanotubes," Phys. Rev. B, Condens. Matter, vol. 74, no. 7, p. 075 415, Aug. 2006.

[30] M. Machon, S. Reich, H. Telg, J. Maultzsch, P. Ordejon, and C. Thomsen, "Strength of radial breathing mode in single-walled carbon nanotubes," Phys. Rev. B, Condens. Matter, vol. 71, no. 3, p. 035 416, 2005.
[31] J. Guo and M. Lundstrom, "Role of phonon scattering in carbon nanotube field-effect transistors," Appl. Phys. Lett., vol. 86, no. 19, p. 193103 , May 2005.

[32] S. O. Koswatta, S. Hasan, M. S. Lundstrom, M. P. Anantram, and D. E. Nikonov, "Ballisticity of nanotube filed-effect transistors: Role of phonon energy and gate bias," Appl. Phys. Lett., vol. 89, no. 2, p. 023 125, Jul. 2006.

[33] S. Hasan, M. A. Alam, and M. Lundstrom, "Simulation of carbon nanotube FETs including hot-phonon and self-heating effects," in IEDM Tech. Dig., 2006, pp. 815-818.

[34] S. O. Koswatta, M. S. Lundstrom, M. P. Anantram, and D. E. Nikonov, "Simulation of phonon-assisted band-to-band tunneling in carbon nanotube field-effect transistors," Appl. Phys. Lett., vol. 87, no. 25, p. 253 107, Dec. 2005.

[35] S. O. Koswatta, M. S. Lundstrom, and D. E. Nikonov, "Band-to-band tunneling in a carbon nanotube metal-oxide-semiconductor field-effect transistor is dominated by phonon assisted tunneling," Nano Lett., vol. 7, no. 5, pp. 1160-1164, 2007.

[36] S. O. Koswatta, D. E. Nikonov, and M. S. Lundstrom, "Computational study of carbon nanotube p-i-n tunnel FETs," in IEDM Tech. Dig., 2005, pp. 518-521.

[37] S. Datta, Quantum Transport: Atom to Transistor. Cambridge, U.K. Cambridge Univ. Press, 2005.

[38] R. Lake, G. Klimeck, R. C. Bowen, and D. Jovanovic, "Single and multiband modeling of quantum electron transport through layered semiconductor devices," J. Appl. Phys., vol. 81, no. 12, pp. 7845-7869, Jun. 1997.

[39] M. P. Anantram, M. S. Lundstrom, and D. E. Nikonov, Modeling of Nanoscale Devices. [Online]. Available: http://arxiv.org/abs/cond-mat/ 0610247

[40] E. V. Anda and F. Flores, "The role of inelastic scattering in tunneling heterostructures," J. Phys.: Condens. Matter, vol. 3, pp. 9087-9101, 1991.

[41] R. Lake, G. Klimeck, and S. Datta, "Rate equations from the Keldysh formalism applied to the phonon peak in resonant tunneling diodes," Phys. Rev. B, Condens. Matter, vol. 47, no. 11, pp. 6427-6438, Mar. 1993.

[42] M. Paulsson, T. Fredericksen, and M. Brandbyge, "Modeling inelastic phonon scattering in atomic- and molecular-wire junctions," Phys. Rev. B, Condens. Matter, vol. 72, no. 20, p. 201 101, Nov. 2005.

[43] A. Pecchia, A. di Carlo, A. Gagliardi, T. A. Niehaus, and T. Frauehnaim, "Atomistic simulation of the electronic transport in organic nanostructures: Electron-phonon and electron-electron interactions," J. Comput. Electron., vol. 4, no. 1/2, pp. 79-82, 2005.

[44] S. Jin, Y. J. Park, and H. S. Min, "A three-dimensional simulation of quantum transport in silicon nanowire transistor in the presence of electron-phonon interactions," J. Appl. Phys., vol. 99, no. 12, pp. $123719.1-123719.10,2006$

[45] S. Jin, "Modeling of quantum transport in nano-sclae MOSFET devices," Ph.D. dissertation, Seoul Nat. Univ., Seoul, Korea, 2006. [Online]. Available: http://www-unix.ecs.umass.edu/ sjin/dissertation.pdf

[46] J. Guo, "A quantum-mechanical treatment of phonon scattering in carbon nanotube transistors," J. Appl. Phys., vol. 98, no. 6, pp. 063519 1-063 519-6, Sep. 2005.

[47] M. Pourfath, H. Kosina, and S. Selberherr, "Rigorous modeling of carbon nanotube transistors," J. Phys.: Conf. Ser, vol. 38, no. 1, pp. 29-32, May 2006.

[48] J. Guo, S. Datta, M. Lundstrom, and M. P. Anantram, "Towards multiscale modeling of carbon nanotube transistors," Int. J. Multiscale Comput. Eng., vol. 2, p. 60, 2004. e-print cond-mat/0312551.

[49] G. Zhang et al., "Selective etching of metallic carbon nanotubes by gasphase reaction," Science, vol. 314, no. 5801, pp. 974-977, Nov. 2006.

[50] R. Venugopal, Z. Ren, S. Datta, M. S. Lundstrom, and D. Jovanovic, "Simulating quantum transport in nanoscale transistors: Real versus modespace approaches," J. Appl. Phys., vol. 92, no. 7, pp. 3730-3739, 2002.

[51] A. Svizhenko and M. P. Anantram, "Role of scattering in nanotransistors," IEEE Trans. Electron Devices, vol. 50, no. 6, pp. 1459-1466, Jun. 2003.

[52] A. Svizhenko and M. P. Anantram, "Effect of scattering and contacts on current and electrostatics in carbon nanotubes," Phys. Rev. B, Condens. Matter, vol. 72, no. 8, pp. 085 430.1-085 430.10, 2005.

[53] M. Lundstrom, Fundamentals of Carrier Transport, 2nd ed. Cambridge, U.K.: Cambridge Univ. Press, 2000.

[54] J. Tersoff, "Schottky barrier height and the continuum gap states," Phys. Rev. Lett., vol. 52, no. 6, pp. 465-468, Feb. 1984.

[55] Z. Ren, "Nanoscale MOSFETs: Physics, simulation, and design," Ph.D. dissertation, Purdue Univ., West Lafayette, IN, 2001.

[56] S. Hasan, "Electron phonon interaction in carbon nanotube devices," Ph.D. dissertation, Purdue Univ., West Lafayette, IN, 2007. 
[57] S. O. Koswatta, S. Hasan, M. S. Lundstrom, M. P. Anantram, and D. E. Nikonov. [Online]. Available: http://arxiv.org/abs/cond-mat/ 0702496

[58] W. Gander and W. Gautschi, "Adaptive quadrature-Revisited," BIT Numer. Math., vol. 40, no. 1, pp. 84-101, 2000.

[59] A. Svizhenko, M. P. Anantram, T. R. Govindan, B. Biegel, and R. Venugopal, "Two-dimensional quantum mechanical modeling of nanotransistors," J. Appl. Phys., vol. 91, no. 4, pp. 2343-2354, Feb. 2002.

[60] [Online]. Available: https://www.nanohub.org/resources/1983/

[61] S. O. Koswatta, N. Neophytou, D. Kienle, G. Fiori, and M. S. Lundstrom, "Dependence of dc characteristics of CNT MOSFETs on bandstructure models," IEEE Trans. Nanotechnol., vol. 5, no. 4, pp. 368-372, Jul. 2006.

[62] S. Datta, "A simple kinetic equation for steady state quantum transport," J. Phys.: Condens. Matter, vol. 2, no. 40, pp. 8023-8052, Oct. 1990.

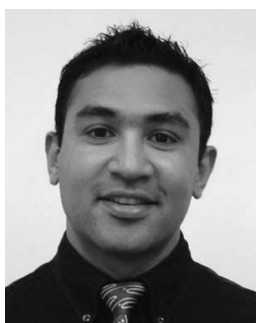

Siyuranga O. Koswatta received the B.S. degree (summa cum laude) in computer engineering from the University of Bridgeport, Bridgeport, CT, in 2002 and the M.S.E.C.E. degree from Purdue University, West Lafayette, IN, in 2004. He is currently working toward the Ph.D. degree in electrical and computer engineering in the School of Electrical and Computer Engineering, Purdue University.

His research interests are mainly in quantum transport and nanoscale device modeling. He has been exploring novel applications and device physics for carbon nanotube transistors.

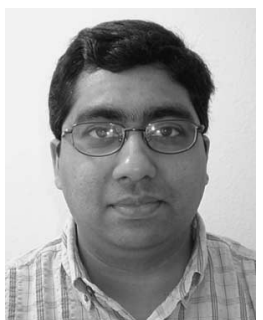

Sayed Hasan received the B.Sc. degree in electri$\mathrm{cal}$ and electronic engineering from the Bangladesh University of Engineering and Technology, Dhaka, Bangladesh, in 1998, and the Ph.D. degree from Purdue University, West Lafayette, IN, in 2007. His doctoral thesis was on electron phonon interaction in carbon nanotube devices. In his doctoral work, he calculated the full-band electron phonon coupling parameters in carbon nanotubes and developed a full-band Boltzmann transport equation solver for electron and phonons in metallic and semi-

conducting tubes.

He is currently with the Device Research Group, Texas Instruments Incorporated, Dallas. His research interest includes coupled electron-phonon and heat transport in metallic and semiconducting carbon nanotubes.

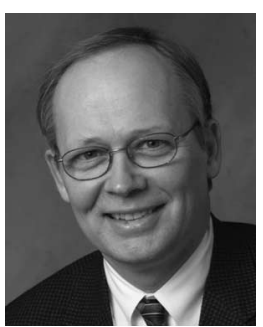

Mark S. Lundstrom (S'72-M'74-SM'80-F'94) received the B.E.E. and M.S.E.E. degrees from the University of Minnesota, Minneapolis, in 1973 and 1974, respectively, and the Ph.D. degree in electrical engineering from Purdue University, West Lafayette, IN, in 1980.

From 1974 to 1977, he was with the HewlettPackard Corporation, Loveland, CO, working on integrated circuit process development and manufacturing support. In 1980, he joined the School of Electrical and Computer Engineering, Purdue University, where he is currently the Don and Carol Scifres Distinguished Professor of Electrical and Computer Engineering and the founding Director of the Network for Computational Nanotechnology. From 1989 to 1993, he was a director of the Optoelectronics Research Center, Purdue University, and, from 1991 to 1994, the Assistant Dean of Engineering. His current research interests include the physics of small electronic devices, especially nanoscale transistors, and carrier transport in semiconductor devices.

Prof. Lundstrom is currently a Distinguished Lecturer of the IEEE Electron Device Society. He is a Fellow of the American Physical Society and the American Association for the Advancement of Science. He received the Frederick Emmons Terman Award from the American Society for Engineering Education in 1992. He, along with his colleague, S. Datta, received the IEEE Cledo Brunetti Award for their work on nanoscale electronic devices and the Semiconductor Research Corporation's Technical Excellence Award in 2002. He received the Semiconductor Industry Association's University Researcher Award for his career contributions to the physics and simulation of semiconductor devices in 2005. Most recently, in 2006, he was the inaugural recipient of the IEEE Electron Device Society's Education Award.

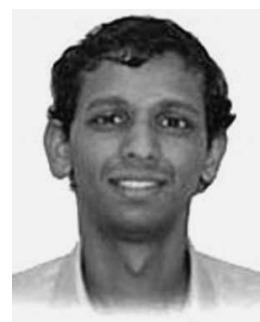

M. P. Anantram received the B.Sc. degree in applied sciences from the PSG College of Technology, Coimbatore, India, in 1986, the M.Sc. degree in physics from the University of Poona, Pune, India, in 1989, and the Ph.D. degree in electrical engineering from Purdue University, West Lafayette, IN, in 1995.

He was the Group Lead for Computational Nanoelectronics with the University Affiliated Research Center, National Aeronautics and Space Administration (NASA) Ames Research Center, where he became a Fellow in 2004. Since May 2006, he has been a Professor with the Nanotechnology Engineering Group, Department of Electrical and Computer Engineering, University of Waterloo, Waterloo, ON, Canada. His group at NASA developed the first 2-D quantum simulator for nanotransistors, and he codeveloped an algorithm to compute the charge and current density in nanodevices. He has also worked extensively on the modeling and prediction of electrical and electromechanical properties of nanostructures. His research encompasses the theory and computational modeling of semiconductor and molecular nanodevices.

Dr. Anantram is an Associate Editor of the IEEE TRANSACTIONS IN NANOTECHNOLOGY and the Education Chair of the IEEE Nanotechnology Council. He is a recipient of best paper awards for his work on nanotransistors and electromechanical properties of nanotubes. He coreceived the Computer Sciences Corporation's highest technical achievement in applied sciences in 2003. He serves on the program and organizing committees of nanotechnology conferences.

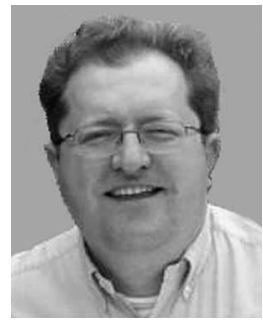

Dmitri E. Nikonov (M'99-SM'06) received the M.S. degree in aeromechanical engineering from Moscow Institute of Physics and Technology, Zhukovsky, Russia, in 1992 and the Ph.D. degree in physics from Texas A\&M University, College Station, in 1996, where he participated in the demonstration of the world's first laser without population inversion.

From 1997 to 1998, he was a Research Engineer and Lecturer with the Department of Electrical and Computer Engineering, University of California at Santa Barbara. He joined the Intel Corporation in 1998 and is currently a Project Manager in the Technology Strategy Group, Santa Clara, CA. He is responsible for managing joint research programs with universities on nanotechnology, optoelectronics, and advanced devices. He was appointed an Adjunct Associate Professor of electrical and computer engineering at Purdue University, West Lafayette, IN, in 2006. He has 33 publications in refereed journals in quantum mechanics, quantum optics, free electron, gas and semiconductor lasers, nanoelectronics, spintronics, and quantum devices simulation. He is the holder of 26 patents in optoelectronics and integrated optics devices.

Dr. Nikonov was a finalist of the Best Doctoral Thesis competition of the American Physical Society in 1997. 